\title{
Application of Ground-Penetrating Radar Broadband Antenna in Underground Detection
}

\author{
ZhangChengke $^{1}$, YuJunping ${ }^{1 *}$, WuJiangpeng ${ }^{1}$, LiZhiqiang $^{1}$, ZhuLiqing $^{1}$ \\ ${ }^{1}$ Jiangxi Transportation Research Institute, Nanchang 330200
}

\begin{abstract}
When GPR is detecting unknown objects underground, different antenna working frequency, different antenna size and different antenna internal structure will affect the final data quality and ultimately affect the detection accuracy of GPR. Therefore, when actualizing and evaluating the uwb signal of GPR electromagnetic wave, the electromagnetic properties of underground medium should be fully considered, and the influence of relevant parameters of GPR antenna on the transmitted and received electromagnetic signals should be analyzed by using numerical analysis method. This paper mainly describes the design characteristics of GPR antenna and antenna array, as well as the types, characteristics and application convenience of antenna array under different positioning purposes of GPR.
\end{abstract}

\section{Introduction}

The use of ground penetrating radar (GPR) for underground detection is a commonly used geophysical method, this method uses electromagnetic pulse signals to map abnormal objects or cavities in the soil, the frequency of electromagnetic waves used is generally between tens of megahertz to several gigahertz. For GPR detection, it is very important to select the appropriate frequency band, it can usually be selected according to the requirements of detection resolution and the depth of underground abnormal objects. The basic principle of GPR detection is the classic radiolocation theory, but compared with general sounding radars, the high-frequency electromagnetic waves emitted by GPR propagate in lossy media, therefore, there is a large dielectric loss. In order to obtain a better resolution within a predetermined depth range, the GPR antenna should have a wider frequency band, a higher amplification factor, and the antenna structure should be compact and suitable. It is best to be carried by a single person, so the ground Radars often use ultra-wideband antennas.

\section{Types and principles of GPR antennas}

Ultra-wideband antennas can be divided into three basic types according to the standard relationship between excitation pulse width and antenna size $\chi=\mathrm{c} \bullet \tau$ (where $\mathrm{c}$ is the speed of light and $\tau$ is the pulse width):

- Traveling wave antenna (size slightly larger than $\chi$ );

- Quasi-resonant antenna (size approximately equal to $\chi$ );

-Elementary antenna (size is much smaller than $\chi$ ).

The usual traveling wave antenna is a TEM horn antenna, which has the advantage of high efficiency and directivity of electromagnetic wave radiation, but its disadvantage is that the overall size is too large, especially when the size of $\mathrm{x}$ is in the range of several decimeters to several meters.

The quasi-resonant antenna has a relatively small size, but compared with the traveling wave antenna, its electromagnetic radiation efficiency and directivity are much inferior. In addition, in a radiated signal, the quasi-resonant antenna can suppress the generation of post-pulse oscillation through complex load effects, as shown in Figure 1.

*Corresponding author:2574544207@qq.com 


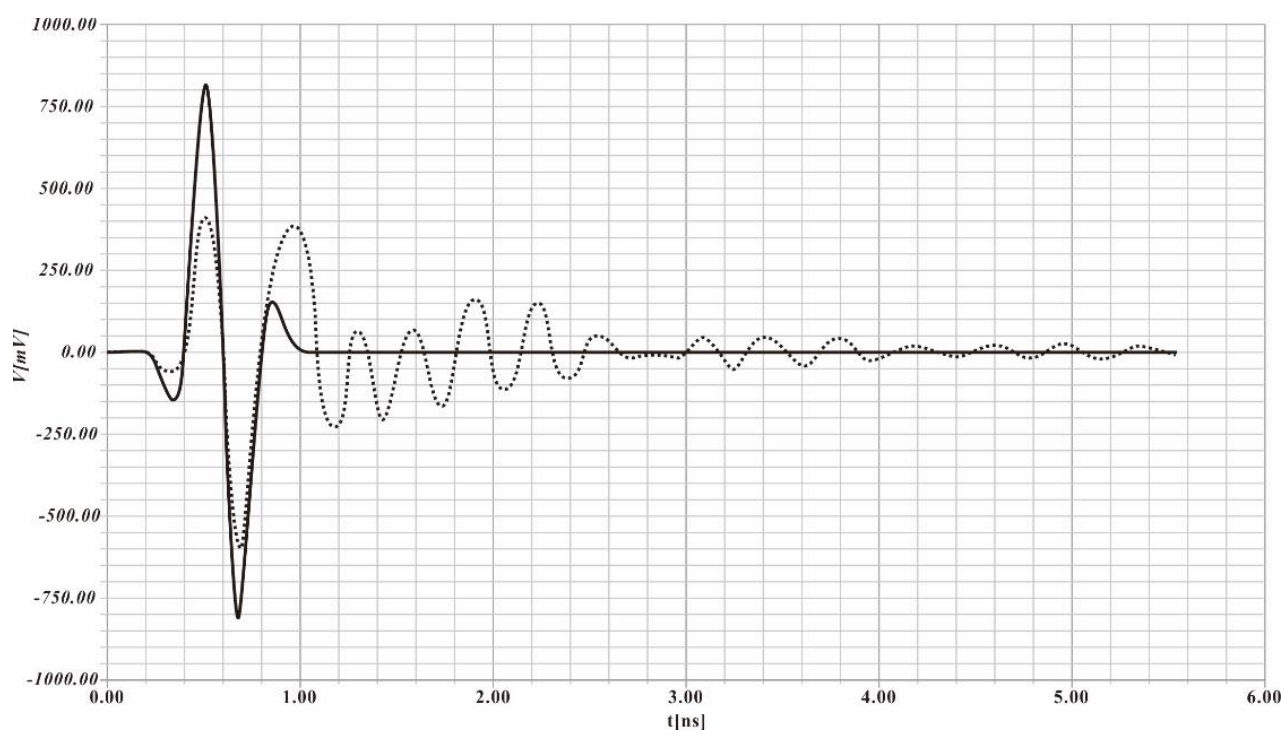

Fig. 1 Ultra-wideband signal input of flat printed biconical antenna

The elementary antenna can radiate and receive ultra-wideband signals, and does not generate pulse after oscillation; the elementary antenna is suitable for use as a basic antenna unit, such as the basic unit of a transceiver antenna array. After design, the antenna array can perform spatial signal processing and The energy of GPR is significantly enhanced ${ }^{[1]}$.

GPR can flexibly use a single transceiver antenna, one receiving antenna and one transmitting antenna, one transmitting antenna and multiple receiving antennas, or multiple transmitting antennas and multiple receiving antennas. The most obvious disadvantage of the more common TEM horn antenna is that it is too large. In contrast, the flat printed biconical antenna has more prominent advantages, that is, low cost, small size, and light weight. Of course, for GPR, greater transmitter power and greater battery capacity are also necessary.

When designing the antenna array, the actual requirements in the application must be considered. First of all, in order to make the electromagnetic wave energy enter the ground as much as possible while using GPR, while reducing the reflection between the electromagnetic wave and the ground, its position is close to the ground surface when used, so the near field effect should be considered when designing the antenna; since the refractive index of soil is greater than that of air, in order to ensure a certain 3D viewing angle, attention should also be paid to limiting the glancing angle effect ${ }^{[2]}$.

When designing the antenna, the main lobe energy should be increased as much as possible, and the side lobe energy should be reduced. But it is quite complicated to achieve this goal. First of all, most of the high-power antennas, the electromagnetic waves radiated by its various components, are inconsistent in frequency and direction. The phase center of the antenna depends on its frequency. When signals of each frequency pass through different paths, the pulse shape will be distorted. Since the feed pulse will be reflected multiple times in its propagation path along the antenna, the input pulse of the antenna will be distorted, which is especially obvious at the edge of the antenna. In the time domain, the effect of the accumulation of multiple reflections will cause the echo pulse to appear obvious tail oscillation. The reason for this phenomenon is that the antenna is essentially a resonant structure with high quality factor $\mathrm{Q}$ and low passband, which is not enough to maintain a complete pulse signal band. Figure 1 shows the time-domain response results of the fed pulse signal in the bow tie antenna (that is, the planar printed biconical antenna). The signal frequency range is between 2 and $5 \mathrm{GHz}$. The result is calculated by the Galerkin method. The dielectric constant of the soil layer is set to 6 .

As shown in Figure 1, the solid line is the input of the UWB signal in the antenna, and the dashed line is the received radiation pulse. The corresponding $\underset{\mathbf{S}(\mathrm{f})}{\operatorname{frequency}}$ spectrum is shown in Figure 2.

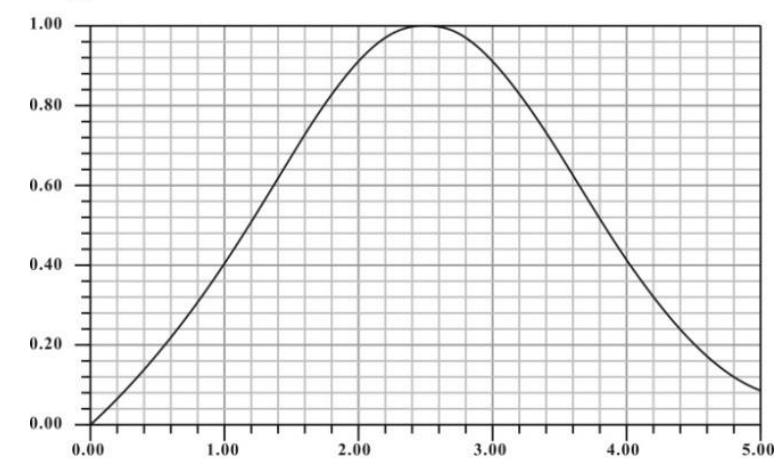

$(\mathrm{Hz})$

Fig. 2 Normalized frequency spectrum of ultra-wideband signal

\section{The influence of different antenna arrays on the measured efficiency}

Corresponding antenna arrays can be arranged in different types of GPR to improve the efficiency of actual measurement. There are currently two methods for the design of antenna array data. The first method is to connect discrete antenna array elements with a controller, and the data processing of each element is 
independent. The second type is a new type of antenna array system developed in recent years. The system exhibits stronger conversion/phase change capabilities and uses synthetic aperture radar algorithms.

The ability of a single antenna array element, that is, a dipole, in gain amplification is limited. The application of antenna arrays can effectively increase the gain and clearly show the directivity of the radar image. Now the point-to-point mode and the collinear mode are used to consider the direction of the object relative to the axis of the antenna array. The expressions $\mathrm{P}(\theta, \varphi)$ of the radiation intensity of the two modes are shown in equations (1) and (2), assuming that all The amplitude and phase of the excitation of the antenna array elements are the same (that is, the equidistant antenna array):

$$
P(\theta, \varphi)=15 \pi\left(\frac{I \Delta l}{\pi}\right)^{2} \frac{\sin ^{2}\left[\frac{N \pi d}{\lambda} \cos \theta\right]}{\sin ^{2}\left[\frac{\pi d}{\lambda} \cos \theta\right]}\left(1-\sin ^{2} \theta \cos ^{2} \theta\right)
$$

$$
P(\theta, \varphi)=15 \pi\left(\frac{I \Delta l}{\pi}\right)^{2} \frac{\sin ^{2}\left[\frac{N \pi d}{\lambda} \cos \theta\right]}{\sin ^{2}\left[\frac{\pi d}{\lambda} \cos \theta\right]}
$$

In the above formula, $\theta$ is the angle (deg), $\lambda$ is the wavelength $(\mathrm{m}), \mathrm{N}$ is the number of radiation elements, $\varphi$ is the phase delay between the radiation elements, $d$ is the distance between the elements, I is the current, and $\Delta \mathrm{l}$ is The incremental path difference of the antenna element.

The array element spacing has a great influence on the frequency multiplier of the antenna array and the upper bound of the actual frequency band of the antenna. Larger element spacing can increase antenna gain. However, in order to reduce the diffraction side lobes, the element spacing of the antenna array should be less than $\lambda / 2$. If there is a large diffracted sidelobe, the gain of the antenna will decrease sharply ${ }^{[3]}$

As shown in Figure 3, at a height of $20 \lambda$ from the plane, the radiated power of the 5-element antenna array and the 11-element antenna array are compared, and the distance between the array elements is set as $\lambda$. You can clearly see the effect of the increase in the number of antenna elements on the gain of the antenna and the suppression of side lobes. Usually the antenna array has a common feeder circuit to provide input signal or output received power for the excitation of the array element. The beam can be formed by setting a certain phase of the array element. In addition, the phase of the array element is usually fixed. Of course, more complex methods can be used, such as adjusting the beam and null mode constraints, and controlling the phase of the array element.

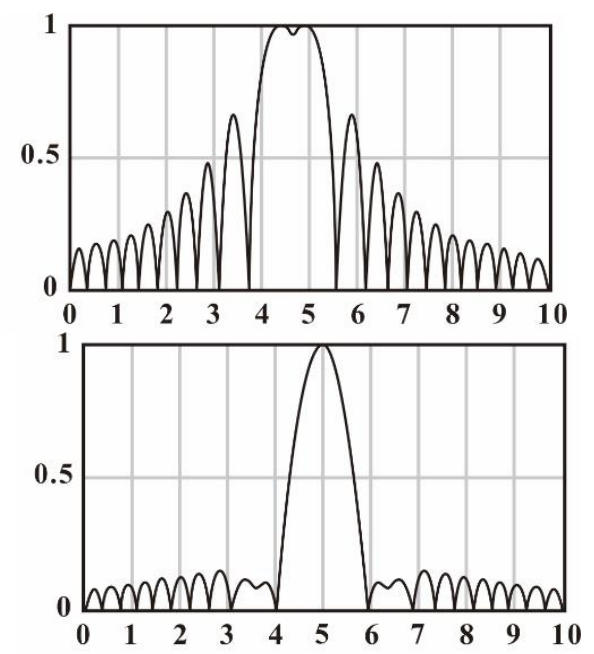

Fig. 3 Comparison of radiated power of 5-element antenna array (up)and 11-element antenna array (down)

The transceiver antenna of the two-dimensional antenna array can be used to obtain the dynamic image of the area under the antenna array. When performing broadband energy scanning, the use of multiple transmitting antenna radiation has a significant advantage. Moreover, multiple receiving antennas can detect the background dissipated energy and achieve noise suppression by synchronizing multiple radar pulses in the time domain.

The independent control system for the transmission and line gates of each transceiver antenna concentrates the radar positioning energy on one point, and then constantly moves the point to scan the area under the antenna array as shown in Figure 4. The differential delay between antenna elements can control the pulse sequence in the time domain, and the beam position can also be adjusted. It should be noted that the delay between the elements is controlled by the maximum equivalent distance between the elements ${ }^{[4]}$.

All antenna array systems are designed to fully obtain image information of unknown objects. The basic condition for achieving this goal is to accurately design and adjust the position of the array elements. An inertial navigation system based on a differential global positioning system (GPS) can be a very effective tool.

When the antenna array is above the ground, it is necessary to correct the influence of changes in the surface terrain. Of course, in order to improve the accuracy, the influence of the distance between the antenna elements should also be considered. Of course, the antenna array should also be large enough, because the density between antenna array elements is closely related to the resolution that the antenna can achieve ${ }^{[2]}$. 


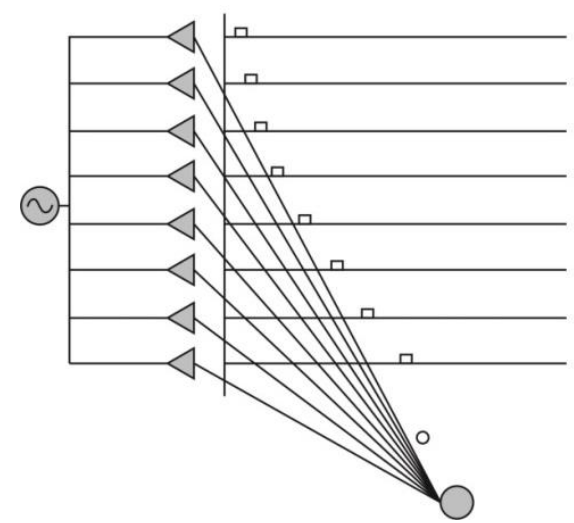

Fig. 4 Adjusting the antenna beam using the time delay of the element radiation

\section{Conclusion}

The antenna system for detecting hidden objects in the underground is classified according to the working frequency range, the size of the antenna, and the internal structure of the antenna. When calculating and analyzing ultra-wideband signals transmitted underground, it is necessary to fully consider the electromagnetic properties of different media.

The numerical calculation method in the time domain can effectively analyze the influence of the geometrical and electrical parameters of the antenna on the radiation and received pulse signals. To be precise, it is its effect on the oscillation after the electromagnetic pulse, so that we can maximize the detection accuracy.

\section{Acknowledgment}

This research was financially supported by the Science and technology project of Jiangxi Provincial Transportation Department(2018H0042), Science and technology project of Jiangxi Provincial Transportation Department(2016H0025), Projects funded by Jiangxi Youth Science Foundation(20181BAB216028).

\section{References}

1. JOL, Harry M. (ed.). Ground penetrating radar theory and applications. elsevier, 2008.

2. ALLEN, Ben, et al. (ed.). Ultra-Wideband Antennas and Propagation: For Communications, Radar and Imaging. John Wiley \& Sons, 2006.

3. DANIELS, David J. EM detection of concealed targets. John Wiley \& Sons, 2009.

4. DANIELS, David J. (ed.). Ground penetrating radar. Iet, 2004. 